\title{
Optimal Minimum Wage in a Competitive Economy: An Alternative Modelling Approach*
}

\author{
Arantza Gorostiaga \\ Universidad del País Vasco
}

\author{
Juan F. Rubio-Ramírez \\ Duke University
}

October, 2006

\begin{abstract}
This paper analyzes whether a minimum wage can be an optimal redistribution policy when distorting taxes and lump-sum transfers are also available in a competitive economy. We build a static general equilibrium model with a Ramsey planner making decisions on taxes, transfers, and minimum wage levels. Workers are assumed to differ only in their productivity. We find that optimal redistribution may imply the use of a minimum wage. The key factor driving our results is the reaction of the demand for low skilled labor to the minimum wage law. Hence, an optimal minimum wage appears to be most likely when low skilled households are scarce, the complementarity between the two types of workers is large or the difference in productivity is small. The main contribution of the paper is a modelling approach that allows us to adopt analysis and solution techniques widely used in recent public finance research. Moreover, this modelling strategy is flexible enough to allow for potential extensions to include dynamics into the model.
\end{abstract}

Keywords: redistribution policy, minimum wage, Ramsey problem JEL: E62, H21

\footnotetext{
*Corresponding Author: Arantza Gorostiaga, Universidad del País Vasco, Departamento de Fundamentos del Análisis Económico II, Avenida Lehendakari Aguirre 83, 48015, Bilbao, Spain. E-mail: arantza.gorostiaga@ehu.es. We thank participants at 2004 SED Conference for useful comments. Financial support from Fundación Ramón Areces, Fundación BBVA (1/BBVA 00044.321-15466/2002) and SEC200302510/ECO is acknowledged.
} 


\section{Introduction}

This paper analyzes whether a minimum wage can be an optimal redistribution policy when distorting taxes and lump-sum transfers are also available in a competitive economy.

Conventional wisdom maintains that a minimum wage is an inefficient policy tool. The main reason behind this argument is the employment loss associated with minimum wage legislation. As labor input becomes more expensive, firms reduce hiring with the consequent welfare loss. However, there are also distortions linked to the use of taxes. Labor income taxation introduces a wedge between the marginal rate of substitution and the marginal rate of transformation between consumption and leisure. As a result, individual labor and consumption decisions are also distorted. Hence, when a minimum wage and labor taxes are considered jointly, the optimal redistribution policy should seek a balance between the inefficiencies due to each of the policy tools.

A lot of research has been carried out into the empirical effect of minimum wage laws. Dolado et al (1996), OECD (1998) and Dickens et al (1999) survey the main results reached by this branch of the literature. But the number of articles that follow a theoretical approach to the issue is much scarcer. ${ }^{1}$ Most of these papers aim to prove that under the assumption of some kind of market failure, the use of a minimum wage can be employment and/or welfare enhancing. This is the case, considering different economic frameworks, of Drèze and Gollier (1993), Rebitzer and Taylor (1995), Cahuc and Michel (1996), Swinnerton (1996), Lang and Kahn (1998), Bhaskar and To (1999) Sampson and Simmons (2002) and Askenazy (2003).

There is also some research that considers the optimal implementation of minimum wage laws to redistribute. Marceau and Boadway (1994) find sufficient conditions for a minimum wage being welfare improving when it is combined with unemployment benefits in an economy with informational asymmetries. In Boadway and Cuff (2001), it is proven that, under private information, non-linear taxes and a minimum wage might increase welfare when typical features of the welfare system are assumed.

Finally, Allen (1987) and Guesnerie and Roberts (1987) are the papers most closely related to ours. They build a general equilibrium model and study whether a minimum wage can improve welfare in an economy with linear or with non-linear taxes on income. Both articles find that minimum wages might be optimally combined with linear taxes. However, when non-linear taxes are considered the minimum wage is no longer optimal.

We build a static general equilibrium model with two types of household, perfectly competitive firms and a government. Households only differ in their labor productivity. Firms use both types of labor as the only inputs to produce a single consumption good. A Ramsey planner implements the optimal redistribution policy, making decisions on taxes, transfers,

\footnotetext{
${ }^{1}$ Dolado et al (2000) goes through the theoretical literature that considers the role of minimum wages as a optimal policy tool.
} 
and minimum wage.

The main contribution of this paper is the modelling approach we present. Our methodology is in the spirit of recent public finance analysis on optimal policies. ${ }^{2}$ We write the problem and characterize the set of equilibria in such a way that the primal approach to optimal policy design can be used. Moreover, this modelling strategy is flexible enough to allow for potential extensions to include dynamics and capital accumulation into the model.

Setting a framework that can be applied to the study of more complex economies is not the only advantage of our approach. In fact, it also allows us to get results that were not available following Allen (1987) or Guesnerie and Roberts (1987). In particular, using our methodology we can compute the optimal size of the minimum wage and characterize the optimal mix of policy tools.

As regards our findings, main results in Allen (1987) or Guesnerie and Roberts (1987) are confirmed by our analysis. We find that, depending upon the economy parameterization, the optimal policy may imply the use of only taxes and transfers, only a minimum wage or the proper combination of both policies. We also learn that the key parameter determining the optimality of the minimum wage is the change in low-skilled labor when this lower bound on wages is enforced. When low-skilled labor demand response to the introduction of a minimum wage is small, the planner finds this policy optimal. This happens when there are few low-skilled households, the complementarity between the two types of input is large or the difference in productivity between one unit of low-skilled labor and one unit of high-skilled labor is small.

The rest of the paper is organized as follows. In section 2 we introduce the model and describe the driving forces of an economy in which the minimum wage binds; we also characterize the Ramsey planner's problem. In section 3 we present the numerical results of the model and study the optimal mix of policies depending on the structural parameterization of the economy. Section 4 concludes.

\footnotetext{
${ }^{2}$ See Lucas and Stokey (1983), Chari and Kehoe (1999), Gorostiaga (2003, 2005) or Schmitt-Grohé and Uribe (2004), just to name a few.
} 


\section{The Model}

We consider a static general equilibrium model. There are two types of household. A measure $\gamma$ of households are high-skilled $(H)$, and $1-\gamma$ are low-skilled $(L)$. A single consumption good is produced. The resource constraint of the economy is:

$$
\gamma c_{H}+(1-\gamma) c_{L}=y
$$

where $y$ is the aggregate production, and $c_{H}$ and $c_{L}$ denote high-skilled and low-skilled consumption respectively.

\subsection{Firms}

The representative firm uses high-skilled and low-skilled workers to produce output. The constant returns to scale production function is characterized as follows:

$$
y=F\left[\gamma\left(1-\ell_{H}\right),(1-\gamma)\left(1-\ell_{L}\right)\right]=\left[\phi\left[\gamma\left(1-\ell_{H}\right)\right]^{\delta}+\left[(1-\gamma)\left(1-\ell_{L}\right)\right]^{\delta}\right]^{1 / \delta}
$$

where $\delta \in(0,1), \phi>1$, and $\left(1-\ell_{H}\right)$ and $\left(1-\ell_{L}\right)$ are high-skilled and low-skilled labor, respectively. Note also that the elasticity of substitution between low-skilled and high-skilled labor is $\frac{1}{1-\delta} \cdot{ }^{3}$ Parameter $\phi$ measures the difference in productivity between high-skilled and low-skilled workers. Firms are price takers, therefore, the inverse labor demands are:

$$
\begin{aligned}
& \omega_{H}=\phi\left[\phi+\left(\frac{1-\gamma}{\gamma}\right)^{\delta}\left(\frac{1-\ell_{L}}{1-\ell_{H}}\right)^{\delta}\right]^{1 / \delta-1} \\
& \omega_{L}=\left[1+\phi\left(\frac{\gamma}{1-\gamma}\right)^{\delta}\left(\frac{1-\ell_{H}}{1-\ell_{L}}\right)^{\delta}\right]^{1 / \delta-1}
\end{aligned}
$$

\subsection{Households}

Type $i \in\{H, L\}$ households derive utility from consumption $\left(c_{i}\right)$ and leisure $\left(\ell_{i}\right)$. The utility function is:

$$
U\left(c_{i}, \ell_{i}\right)
$$

\footnotetext{
${ }^{3}$ With $\delta=1$ we have perfect substitutability between inputs and $\delta=0$ yields a Cobb-Douglas production function.
} 
where $U$ is assumed to be strictly increasing and concave in both arguments. Households are endowed with one unit of time which can be devoted to work $\left(1-\ell_{i}\right)$ or leisure $\left(\ell_{i}\right)$, and face the following budget constraint:

$$
c_{i}=(1-\tau) \omega_{i}\left(1-\ell_{i}\right)+T
$$

where $\omega_{i}$ is wage, $\tau$ is the income tax rate and $T$ is a lump-sum transfer. ${ }^{4}$

\subsection{Minimum Wage}

In addition, there is a statutory minimum wage in this economy. Therefore, in equilibrium, wages must be higher than a legal lower bound, $\omega_{\min }$. When the minimum wage is binding in equilibrium, there is an excess labor supply and the demand side determines labor allocations. Although in this economy there are two types of labor, we consider parameter configurations for which only low-skilled workers may be constrained. Therefore, because of the minimum wage legislation, low-skilled labor supply faces an additional constraint: there is a maximum number of hours, $\overline{S C}_{L},{ }^{5}$ each low-skilled household can allocate to work at the minimum wage $\omega_{\text {min }} \cdot \overline{S C}_{L}$ is equal to the hours of low-skilled labor that the firm demands at the minimum wage in equilibrium. ${ }^{6}$

Therefore, household $i$ 's decision problem is:

$$
\begin{aligned}
& \max _{\left\{c_{i}, \ell_{i}\right\}} U\left(c_{i}, \ell_{i}\right) \\
\text { s.t. } c_{i}= & (1-\tau) \omega_{i}\left(1-\ell_{i}\right)+T \\
\left(1-\ell_{i}\right) \leq & \overline{S C}_{i} \\
& \omega_{i}, \overline{S C}_{i}, \tau, T \text { given }
\end{aligned}
$$

The first order conditions for this problem are:

$$
\begin{aligned}
& -U_{c}\left(c_{i}, \ell_{i}\right)(1-\tau) \omega_{i}+U_{\ell}\left(c_{i}, \ell_{i}\right)+v_{i}=0 \\
& v_{i}\left[\overline{S C}_{i}-\left(1-\ell_{i}\right)\right]=0 \\
& \overline{S C}_{i}-\left(1-\ell_{i}\right) \geq 0 \quad v_{i} \geq 0 \\
& c_{i}=(1-\tau) \omega_{i}\left(1-\ell_{i}\right)+T
\end{aligned}
$$

\footnotetext{
${ }^{4}$ Note that under this tax scheme, the marginal tax rate is constant, but the average tax rate increases with income.

${ }^{5} \overline{S C}_{L}\left(\omega_{\min }\right)$ depends on $\omega_{\min }$. To simplify notation, we do not make this relationship explicit in what follows.

${ }^{6}$ We follow the disequilibrium theory approach to model the effect of minimum wages on the household problem. See Malinvaud (1977).
} 
where $v_{i}$ is the multiplier associated with the quantity constraint on $i$-skilled labor supply. We consider economies where the minimum wage only binds for low-skilled workers, i.e., high-skilled labor will never be constrained. Hence,

$$
\overline{S C}_{H}-\left(1-\ell_{H}\right)>0
$$

\subsection{Government}

The government is a social planner who chooses labor income taxes, lump sum transfers, and the minimum wage so as to maximize welfare. The welfare function we consider is:

$$
W=\varphi \gamma U\left(c_{H}, \ell_{H}\right)+(1-\varphi)(1-\gamma) U\left(c_{L}, \ell_{L}\right),
$$

where $\varphi$ is the weight of high-skilled workers in the welfare function.

Tax revenues are rebated to households through a lump sum transfer. The government budget must be balanced. Hence, her budget constraint can be written as:

$$
T=\tau\left[\gamma\left(1-\ell_{H}\right) \omega_{H}+(1-\gamma)\left(1-\ell_{L}\right) \omega_{L}\right]
$$

\subsection{Equilibrium}

In this economy, an equilibrium is defined as follows:

Definition 1. Given a minimum wage $\omega_{\min }$, allocations $\left(c_{H}, c_{L}, \ell_{H}, \ell_{L}\right)$, taxes and transfers $(\tau, T)$, wages $\left(\omega_{H}, \omega_{L}\right)$ and perceived constraints on the low-skilled labor supply $\overline{S C}_{L}$ constitute an equilibrium if the following conditions are satisfied:

(i) $\left(c_{H}, \ell_{H}\right)$ solves the high-skilled household's decision problem given $\omega_{H}$ and policies $(\tau, T)$.

(ii) $\left(c_{L}, \ell_{L}\right)$ solves the low-skilled household's decision problem given $\omega_{L}$, policies $(\tau, T)$ and $\overline{S C}_{L}$.

(iii) $\left(\ell_{H}, \ell_{L}\right)$ maximizes firm's profits given $\omega_{H}$ and $\omega_{L}$.

(iv) The equilibrium wage $\omega_{L}$ is equal to or higher than $\omega_{\min }$. And $\overline{S C}_{L}$ is the quantity of low-skilled labor demanded at the minimum wage $\omega_{\min }$.

$$
\omega_{L} \geq \omega_{\min }=F_{1-\ell_{L}}\left[\gamma\left(1-\ell_{H}\right),(1-\gamma) \overline{S C}_{L}\right]
$$

(v) The economy resource constraint (1) and government budget constraint (6) hold. 
To gain insight into the main driving forces of the economy, let us describe how equilibrium allocations are determined, given a policy $\left(\omega_{\min }, \tau, T\right)$, when the minimum wage binds. Given the minimum wage, $\omega_{\min }$, the production side determines the equilibrium high-skilled to lowskilled labor ratio, $\frac{1-\ell_{H}}{1-\ell_{L}}\left(\omega_{\min }\right)$, and high-skilled workers' wage, $\omega_{H}\left(\omega_{\min }\right)$. The high-skilled to low-skilled labor ratio is set such that the marginal low-skilled labor productivity equals the minimum wage:

$$
\omega_{\min }=\left[1+\phi\left(\frac{\gamma}{1-\gamma}\right)^{\delta}\left(\frac{1-\ell_{H}}{1-\ell_{L}}\left(\omega_{\min }\right)\right)^{\delta}\right]^{\frac{1}{\delta}-1},
$$

while the high-skilled workers' wage is determined by the high-skilled workers' labor productivity:

$$
\omega_{H}\left(\omega_{\min }\right)=\phi\left[\phi+\left(\frac{1-\gamma}{\gamma}\right)^{\delta}\left(\frac{1}{\frac{1-\ell_{H}}{1-\ell_{L}}\left(\omega_{\min }\right)}\right)^{\delta}\right]^{\frac{1}{\delta}-1} .
$$

Then, households choose how many hours to work and how much to consume. Highskilled households' consumption, $c_{H}$, and leisure, $\ell_{H}$, are such that the following first order conditions are satisfied at the equilibrium wage, $\omega_{H}\left(\omega_{\min }\right)$ :

$$
\begin{gathered}
-U_{c}\left(c_{H}, \ell_{H}\right)(1-\tau) \omega_{H}\left(\omega_{\min }\right)+U_{\ell}\left(c_{H}, \ell_{H}\right)=0, \text { and } \\
c_{H}=(1-\tau) \omega_{H}\left(\omega_{\min }\right)\left(1-\ell_{H}\right)+T .
\end{gathered}
$$

Finally, low-skilled labor allocation, $1-\ell_{L}$, is such that the labor ratio, $\frac{1-\ell_{H}}{1-\ell_{L}}\left(\omega_{\min }\right)$, holds, while low-skilled consumption, $c_{L}$, is such that the corresponding budget constraint is fulfilled.

Therefore, when the minimum wage binds, the production side determines wages and labor composition, while the households' side determines consumption and labor allocations.

As equation (7) indicates, for a given parameterization, the higher the binding minimum wage, the higher the equilibrium high-skilled to low-skilled labor ratio. As low-skilled labor becomes more expensive, firms use it less. At the same time, equation (8) shows that as relatively more high-skilled workers are hired, their marginal productivity and wage decrease.

As regards the effects of the structural parameters in the technology on the reaction of high-skilled to low-skilled labor ratio to a larger minimum wage, as $\delta$, grows, i.e. as inputs become closer substitutes, it is easier to substitute high-skilled workers for low-skilled ones. Therefore, the effect of the introduction of a minimum wage on the high-skilled to low-skilled labor ratio is larger. As $\phi$ increases, high-skilled workers are more productive, and, again, it becomes easier to replace any fixed number of low-skilled workers. Lastly, the larger the measure of high-skilled households, $\gamma$, the more essential low-skilled labor is in the production process and, hence, the effects of a minimum wage legislation on equilibrium low-skilled labor allocations are more limited. 


\subsection{Distortions and Allocations Effects}

In this section, we explain first what the main distortions caused by either a minimum wage or a tax and transfer scheme are. And then we describe the most important effects of each redistribution instrument on allocations. For the sake of simplicity, let us consider each instrument on its own. Hence, when analyzing minimum wage, we will set taxes to zero, and when studying taxes, we will set the minimum wage to zero.

\subsubsection{Distortions}

\section{Minimum Wage}

When the minimum wage binds, two important distortions appear. On the production side, low-skilled labor gets more expensive and firms hire less of it. Thus, there is an employment loss and the composition of inputs becomes distorted. On the consumer side, low-skilled workers are willing to work more hours than the firm finds profitable to hire them for. As a result, low-skilled labor is in excess supply. The following equilibrium inequality shows this effect:

$$
U_{c}\left(c_{L}, \ell_{L}\right) \omega_{\min }>U_{\ell}\left(c_{L}, \ell_{L}\right) .
$$

Taxes and transfers

This case has been widely studied in the literature and the results are well-known. Taxes distort consumers' decisions by introducing a wedge between the marginal rate of substitution and the marginal rate of transformation between consumption and leisure. The labor supply shifts leftwards and a deadweight loss arises. The following equilibrium equation shows the effect:

$$
(1-\tau) \omega_{i}=\frac{U_{\ell}\left(c_{i}, \ell_{i}\right)}{U_{c}\left(c_{i}, \ell_{i}\right)}
$$

for $i \in\{H, L\}$.

\subsubsection{Effects on Allocations}

\section{Minimum Wage}

If a minimum wage is optimal, low-skilled workers are more expensive and firms hire less of them. Numerical exercises show that low-skilled workers devote more time to leisure and consume less than at the competitive equilibrium. The utility gains due to more leisure time are higher than utility losses caused by the consumption drop, and low-skilled agents enjoy larger utility. High-skilled workers see their wage decrease and reduce their consumption. Finally, depending on the sign of the elasticity of labor supply, they may increase or decrease the time they devote to production activities. In any case, high-skilled workers are worse off. 
Actually, the effect of a binding minimum wage on high-skilled and low-skilled utility does not depend on assumed functional forms or on the model parameterization.

Taxes and transfers

As it is the case for the minimum wage policy, when redistribution is implemented through taxes and transfers, high-skilled workers are worse off and low-skilled workers are better off than they were at the competitive equilibrium. Our numerical results show that lowskilled households work less than at the competitive equilibrium. Their consumption increases except for cases in which productivity differentials are very small. High-skilled household consumption decreases. Again, the sign of the elasticity of labor supply makes the effect on hours worked by high-skilled households ambiguous.

\subsection{The Ramsey Problem}

The government is a Ramsey planner. She takes households' reaction to policy announcements into account when solving for the optimal redistribution policy. Our analysis builds on the primal approach to optimal taxation. The planner selects the policy that maximizes the welfare function over equilibrium allocations. In order to solve the Ramsey problem, we eliminate wages, taxes, and transfers from the set of equations that determine an equilibrium using the households and firm first order conditions. The following proposition states that the set of equilibrium allocations can be characterized by five conditions.

Proposition 1. Given a minimum wage $\omega_{\min }$, allocations $\left(c_{H}, c_{L}, \ell_{H}, \ell_{L}\right)$, taxes and transfers $(\tau, T)$, wages $\left(\omega_{H}, \omega_{L}\right)$ and perceived constraints on the low-skilled labor supply $\overline{S C}_{L}$ constitute an equilibrium if and only if the following conditions are satisfied:

$$
\begin{gathered}
U_{c}\left(c_{H}, \ell_{H}\right) c_{H} \omega_{H}-U_{\ell}\left(c_{H}, \ell_{H}\right) \omega_{H}\left(1-\ell_{H}\right)-\left[U_{c}\left(c_{H}, \ell_{H}\right) \omega_{H}-U_{\ell}\left(c_{H}, \ell_{H}\right)\right] y=0, \\
U_{c}\left(c_{L}, \ell_{L}\right) U_{\ell}\left(c_{H}, \ell_{H}\right) \omega_{L}-U_{c}\left(c_{H}, \ell_{H}\right) U_{\ell}\left(c_{L}, \ell_{L}\right) \omega_{H} \geq 0, \\
{\left[U_{c}\left(c_{L}, \ell_{L}\right) U_{\ell}\left(c_{H}, \ell_{H}\right) \omega_{L}-U_{c}\left(c_{H}, \ell_{H}\right) U_{\ell}\left(c_{L}, \ell_{L}\right) \omega_{H}\right]\left[S C_{L}-\left(1-\ell_{L}\right)\right]=0,} \\
\overline{S C}_{L}-\left(1-\ell_{L}\right) \geq 0,
\end{gathered}
$$

and

$$
(1-\gamma) c_{L}+\gamma c_{H}=y
$$

with

$$
\begin{aligned}
\omega_{L} & =F_{1-\ell_{L}}\left[\gamma\left(1-\ell_{H}\right),(1-\gamma)\left(1-\ell_{L}\right)\right], \\
\omega_{H} & =F_{1-\ell_{H}}\left[\gamma\left(1-\ell_{H}\right),(1-\gamma)\left(1-\ell_{L}\right)\right], \\
y & =F\left[\gamma\left(1-\ell_{H}\right),(1-\gamma)\left(1-\ell_{L}\right)\right] .
\end{aligned}
$$


The proof of the proposition is technical and can be found in the appendix.

Equations (9) and (10) are what the literature calls implementability constraints. They are the result of substituting out prices and policies in the high-skilled and low-skilled budget constraints using household problem's first order conditions. Equations (10), (11), and (12) encompass the possibility of finding constrained low-skilled labor. Finally, equation (13) is the market clearing condition.

This new characterization of the equilibrium allows us to define the set of equilibria as a function of allocations, $\left(c_{L}, c_{H}, \ell_{L}, \ell_{H}, \overline{S C}_{L}\right)$, instead of as a function of prices, $\left(\omega_{H}, \omega_{L}\right)$, and policies, $\left(\omega_{\min }, \tau, T\right)$. Therefore, the primal approach to optimal policy taxation can be applied.

The Ramsey problem can be written as the maximization of the welfare function (5) subject to the set of equations that, as proven in Proposition 1 , characterize the set of equilibria. Taking derivatives with respect to $c_{L}, c_{H}, \ell_{L}, \ell_{H}$ and $\overline{S C}_{L}$ we obtain the system of equations that have to be solved to find the optimal allocation. ${ }^{7}$.

\section{Results}

The aim of the paper is to analyze the optimal mix of minimum wage, taxes and transfers in order to maximize social welfare. In this section we numerically compute the optimal policy. We perform several numerical exercises to study how different parameterizations affect the optimal redistribution policy.

For the numerical exercises we assume a CES functional form for the utility function:

$$
U(c, \ell)=\frac{c^{1-\sigma}}{1-\sigma}+\frac{\ell^{1-\sigma}}{1-\sigma}
$$

We do not calibrate. We simply consider a widely used range of values for each parameter. The parameter controlling the elasticity of labor supply, $\sigma$, varies between 0.5 and 3 . The parameter setting the relative productivity of high-skilled workers, $\phi$, ranges from 1.15 to 2 . The parameter affecting the elasticity of substitution between high and low-skilled workers, $\delta$, extends from 0.2 to 0.9 . The measure of high-skilled households, $\gamma$, varies from 0.2 to 0.8 . Finally, the social planner attaches the same weight to the utility of both types of household, that is, $\varphi$ is set equal to 0.5 .

We examine the effect on minimum wages, taxes and transfers of moving pairs of parameters while keeping the others fixed at a benchmark parameterization (see Table 1).

\footnotetext{
${ }^{7}$ The system of first order conditions and the procedure used to find numerically optimal allocations can be found in Appendix B.
} 


\section{Table 1: Benchmark Parameterization}

\begin{tabular}{|c|c|c|c|c|}
\hline$\sigma$ & $\phi$ & $\delta$ & $\gamma$ & $\varphi$ \\
\hline 2 & 1.5 & 0.3 & 0.5 & 0.5 \\
\hline
\end{tabular}

We find that, depending on the structural parameters, it may be optimal to use only taxes and transfers, only minimum wages or both to implement the redistribution policy.

In general terms, we find that for parameterizations that imply high labor productivity inequality, taxes and transfers are a better redistribution tool. As labor productivity gets more equally distributed, minimum wage becomes optimal.

Why does minimum wage appear to be optimal when labor productivity inequality decreases? It can be proven straightforwardly that the low to high-skilled labor productivity ratio is:

$$
\frac{\omega_{L}}{\omega_{H}}=\frac{1}{\phi}\left[\left(\frac{\gamma}{1-\gamma}\right)\left(\frac{1-\ell_{H}}{1-\ell_{L}}\right)\right]^{1-\delta}
$$

Since we only consider the optimal policy for parameter values such that $\frac{\omega_{L}}{\omega_{H}}$ is smaller than one, as the ratio above increases differences in labor productivity decrease. Therefore, labor productivity inequality falls when low-skilled households are a scarce resource $(\gamma$ large), more complementary to high-skilled workers $(\delta$ small $)$, or similarly productive to them ( $\phi$ close to one).

When low-skilled workers are relatively less productive and their wage increases, the firm finds profitable to substitute a larger portion of low-skilled labor by high-skilled labor. Therefore, a binding minimum wage causes a large reduction in both the hiring of lowskilled workers and the high-skilled wage. Consequently, the fall in both types of households' consumption is likely to be higher. The effects on social welfare are twofold. On the one hand, the low-skilled utility loss caused by the drop in consumption increases and could even outweigh the gain due to the larger leisure enjoyed. On the other hand, drops in high-skilled consumption result in larger utility decreases. Therefore, the probability is that a minimum wage will not be welfare improving.

Figures 1 to 6 display the optimal mix of policies depending on pairs of parameters. The numerical exercises yield five different possible combinations of optimal policies: (1) only taxes and transfers, (2) taxes, transfers and minimum wage, (3) only minimum wage, (4) minimum wage with negative taxes and transfers, and (5) no redistribution policy. ${ }^{8}$

For example in Figure 1 when $\gamma$ equals 0.3 and $\sigma$ equals 1, the optimal policy considers only taxes and transfers, while when $\gamma$ equals 0.5 and $\sigma$ equals 1 , it is optimal to use taxes,

\footnotetext{
${ }^{8}$ This last case also includes parameterizations such that low skilled income is higher than high skilled income at the competitive equilibrium.
} 
transfers and minimum wage. Finally, when $\gamma$ equals 0.75 and $\sigma$ equals 1.5 , no redistribution policy is found to be optimal.

Figures 1 to 3 exhibit the optimal mix of policies for different values of the measure of high-skilled households, $\gamma$, combined with different values of the remaining parameters. In all cases, as $\gamma$ grows, or as the measure of low-skilled people decreases, the minimum wage appears as an optimal redistribution policy, regardless of the values of the rest of the parameters. When the measure of high-skilled people is low, only taxes are used. As we consider economies with more high-skilled people, a combination of taxes and minimum wage becomes optimal. There is always a narrow region where only a minimum wage is optimal, but as $\gamma$ approaches one, (negative) taxes arise again. Finally, for values of $\gamma$ very close to one, the optimal policy is no policy.

As low-skilled workers become scarce, they turn out to be more productive (labor productivity inequality is reduced), and the firm does not reduce the use of such workers even when a minimum wage is introduced. When $\gamma$ is close to one, both workers are so similar that redistribution is not optimal.

Let us now analyze how the optimal mix of policies changes when the elasticity of substitution between inputs, $\delta$, varies. This is done in Figures 3 to 5 . In all cases, as $\delta$ decreases, or as the inputs become more complementary, it is optimal to use a minimum wage for redistribution. As before, this effect is true independently of the values for the rest of the parameters. When low and high-skilled labor are complements, $\delta$ low, it is harder for firms to substitute high-skilled for low-skilled labor, so the introduction of a minimum wage has a small effect on the relative hiring of low-skilled labor.

Figures 2, 5 and 6 show the effects of $\phi$ on the optimal redistribution policy mix. In all cases, as $\phi$ approaches one, or as differences in productivity of one unit of low-skilled and one unit of high-skilled labor are smaller, a minimum wage has a smaller negative effect on the low-skilled employment, consumption and welfare. Hence, it is optimal to use a minimum wage for redistribution. Again, this effect is true no matter what values the rest of the parameters take.

Figures 1, 4, and 6 display the optimal redistribution policy tools for different values of $\sigma$. This is the preference parameter that determines the size and sign of the elasticity of labor supply. As can be observed in the figures, the effects of the rest of the parameters cancel out any possible effect of $\sigma$ on the optimal policy. This is due to the fact that changes in these parameters affect the elasticity of labor supply of both types of household symmetrically. Therefore, any change in $\sigma$ does not directly affect the labor productivity ratio of the economy.

Finally, it should be noted that workers in our model are never unemployed as a result of the minimum wage law. We assume that when a binding minimum wage causes low skilled labor hiring to reduce, workers will work fewer hours than they are willing to at the market wage. That is, workers will be only under-employed. As Allen (1987) points out, if the 
reduction of the hiring falls only on a specific group of low-skilled workers, the minimum wage policy is less likely to be optimal.

\section{Concluding Remarks}

In this paper we have studied the optimality of a minimum wage as a redistribution policy tool in a competitive economy where distorting taxes and lump-sum transfers are also available. We have modelled the economy to be able to use the primal approach to optimal policy. We have characterized the equilibrium and we have computed the proper combination of policies for different values of the structural parameters of the model.

We have shown that labor productivity differentials are a crucial factor in explaining the optimal policy mix. Whenever productivity inequality decreases, the minimum wage appears to be optimal on its own or properly combined with a tax-transfer scheme. In our economy, low-skilled workers' relative productivity is larger when low-skilled workers are scarce, when low-skilled labor is a good complement to high-skilled labor, or when the difference in productivity of one unit of any input is small. In that case, a statutory lower bound on wages does not greatly alter low-skilled labor allocation in equilibrium. Thus, larger welfare gains due to a minimum wage make its use to implement the optimal redistribution policy more likely.

There are several additional issues that we have not considered and that we leave for future analysis. First, our model is static and deterministic. Hence, a natural extension is to consider a dynamic and/or stochastic version of the economy. Our guess is that a minimum wage might be a useful policy as a buffer to soften the effects of shocks. Second, since we only study a static version, we do not consider capital. A binding minimum wage changes input productivity and could have an important impact on capital accumulation and growth. As a result, the optimal policy design might also be different. One of the main advantages of our modelling approach is that these extensions can be carried out in a similar theoretical framework and that the recent public finance literature has developed the required numerical techniques to solve these models to find optimal policies. 


\section{Appendix A}

Proof of Proposition 1. First, we prove that a competitive equilibrium implies equations (9)-(13).

Notice that conditions (12) and (13) are fulfilled by definition of a competitive equilibrium.

From the first order conditions of the households' problems the following equations should be satisfied in equilibrium:

$$
\begin{gathered}
-U_{c}\left(c_{L}, \ell_{L}\right)(1-\tau) \omega_{L}+U_{\ell}\left(c_{L}, \ell_{L}\right)+v_{L}=0 \\
v_{L}\left[\overline{S C}_{L}-\left(1-\ell_{L}\right)\right]=0 \\
\overline{S C}_{L}-\left(1-\ell_{L}\right) \geq 0 \quad \nu \geq 0 \\
c_{L}=(1-\tau) \omega_{L}\left(1-\ell_{L}\right)+T \\
-U_{c}\left(c_{H}, \ell_{H}\right)(1-\tau) \omega_{H}+U_{\ell}\left(c_{H}, \ell_{H}\right)=0 \Rightarrow \tau=1-\frac{U_{\ell}\left(c_{H}, \ell_{H}\right)}{U_{c}\left(c_{H}, \ell_{H}\right) \omega_{H}} \\
c_{H}=(1-\tau) \omega_{H}\left(1-\ell_{H}\right)+T
\end{gathered}
$$

Substituting (18) into (14) and solving for the Kuhn-Tucker multiplier $v_{L}$, we get:

$$
v_{L}=\frac{U_{c}\left(c_{L}, \ell_{L}\right) U_{\ell}\left(c_{H}, \ell_{H}\right) \omega_{L}-U_{c}\left(c_{H}, \ell_{H}\right) U_{\ell}\left(c_{L}, \ell_{L}\right) \omega_{H}}{U_{c}\left(c_{H}, \ell_{H}\right) \omega_{H}} \geq 0
$$

This result and expressions (15) and (16) imply equation (11):

$$
\left[U_{c}\left(c_{L}, \ell_{L}\right) U_{\ell}\left(c_{H}, \ell_{H}\right) \omega_{L}-U_{c}\left(c_{H}, \ell_{H}\right) U_{\ell}\left(c_{L}, \ell_{L}\right) \omega_{H}\right]\left[\overline{S C}_{L}-\left(1-\ell_{L}\right)\right]=0,
$$

and equation (10):

$$
U_{c}\left(c_{L}, \ell_{L}\right) U_{\ell}\left(c_{H}, \ell_{H}\right) \omega_{L}-U_{c}\left(c_{H}, \ell_{H}\right) U_{\ell}\left(c_{L}, \ell_{L}\right) \omega_{H} \geq 0 .
$$

Finally, from equation (18), the high-skilled consumer budget constraint (19) and the government budget constraint (6) we get equation (9):

$$
U_{c}\left(c_{H}, \ell_{H}\right) c_{H} \omega_{H}-U_{\ell}\left(c_{H}, \ell_{H}\right) \omega_{H}\left(1-\ell_{H}\right)-\left[U_{c}\left(c_{H}, \ell_{H}\right) \omega_{H}-U_{\ell}\left(c_{H}, \ell_{H}\right)\right] y=0
$$

Now we will prove that for any set of allocations $c_{L}, c_{H}, \ell_{L}$ and $\ell_{H}$ fulfilling equations (9)(13) we can construct a process for policies $\left\{\tau, T, \omega_{\min }\right\}$ and prices $\left\{\omega_{L}, \omega_{H}\right\}$ that constitute a competitive equilibrium. 
Wage rates are determined from allocations $\ell_{L}$ and $\ell_{H}$ and equations:

$$
\begin{aligned}
\omega_{L} & =F_{1-\ell_{L}}\left[\gamma\left(1-\ell_{H}\right),(1-\gamma)\left(1-\ell_{L}\right)\right] \\
\omega_{H} & =F_{1-\ell_{H}}\left[\gamma\left(1-\ell_{H}\right),(1-\gamma)\left(1-\ell_{L}\right)\right]
\end{aligned}
$$

The labor income tax rate and transfers will be pinned down by:

$$
\begin{gathered}
\tau=1-\frac{U_{\ell}\left(c_{H}, \ell_{H}\right)}{U_{c}\left(c_{H}, \ell_{H}\right) \omega_{H}} \\
T=\tau y
\end{gathered}
$$

When the following expression

$$
U_{c}\left(c_{L}, \ell_{L}\right) U_{\ell}\left(c_{H}, \ell_{H}\right) \omega_{L}-U_{c}\left(c_{H}, \ell_{H}\right) U_{\ell}\left(c_{L}, \ell_{L}\right) \omega_{H}
$$

is strictly positive, $\overline{S C}_{L}=1-\ell_{L}$, that is, the minimum wage law is binding and therefore,

$$
\omega_{\min }=F_{1-\ell_{L}}\left[\gamma\left(1-\ell_{H}\right),(1-\gamma) \overline{S C}_{L}\right]
$$

When it is equal to zero, then the minimum wage is not binding $\left(\overline{S C}_{L}>1-\ell_{L}\right)$ and there are multiple values for $\omega_{\min }$ satisfying the required conditions:

$$
\omega_{\min }<F_{1-\ell_{L}}\left[\gamma\left(1-\ell_{H}\right),(1-\gamma) \overline{S C}_{L}\right]
$$

Equation (15) holds if $v_{L}$ is defined as,

$$
v_{L}=\frac{U_{c}\left(c_{L}, \ell_{L}\right) U_{\ell}\left(c_{H}, \ell_{H}\right) \omega_{L}-U_{c}\left(c_{H}, \ell_{H}\right) U_{\ell}\left(c_{L}, \ell_{L}\right) \omega_{H}}{U_{c}\left(c_{H}, \ell_{H}\right) \omega_{H}}
$$

Finally, we prove that allocations and prices are such that households' budget constraints are fulfilled. From (9), considering the way in which we have defined and computed tax rates and transfers, we have:

$$
U_{c}\left(c_{H}, \ell_{H}\right) c_{H} \omega_{H}-U_{\ell}\left(c_{H}, \ell_{H}\right) \omega_{H}\left(1-\ell_{H}\right)-\left[U_{c}\left(c_{H}, \ell_{H}\right) \omega_{H}-U_{\ell}\left(c_{H}, \ell_{H}\right)\right] y=0,
$$

which implies

$$
U_{c}\left(c_{H}, \ell_{H}\right) c_{H} \omega_{H}-U_{\ell}\left(c_{H}, \ell_{H}\right) \omega_{H}\left(1-\ell_{H}\right)-U_{c}\left(c_{H}, \ell_{H}\right) \omega_{H} \tau y=0,
$$

or

$$
c_{H}-\frac{U_{\ell}\left(c_{H}, \ell_{H}\right)}{U_{c}\left(c_{H}, \ell_{H}\right)}\left(1-\ell_{H}\right)-T=0,
$$

which is equivalent to

$$
c_{H}-(1-\tau) \omega_{H}\left(1-\ell_{H}\right)-T=0 .
$$

Once we have proven that the high-skilled household budget constraint holds, this restriction, the resource constraint (13) and the government budget constraint (6) guarantee that the low-skilled budget constraint is also satisfied. 


\section{Appendix B}

This appendix presents the Ramsey problem first order conditions that characterize the optimal policy. Besides it is also described the procedure used to solve the system of equations.

The Ramsey problem can be written as:

$$
\max _{\left\{c_{L}, c_{H}, \ell_{L}, \ell_{H}, \overline{S C}_{L}\right\}} \varphi \gamma U\left(c_{H}, \ell_{H}\right)+(1-\varphi)(1-\gamma) U\left(c_{L}, \ell_{L}\right)
$$

s.t.

$$
\begin{gathered}
U_{c}\left(c_{H}, \ell_{H}\right) c_{H} \omega_{H}-U_{\ell}\left(c_{H}, \ell_{H}\right) \omega_{H}\left(1-\ell_{H}\right)-\left[U_{c}\left(c_{H}, \ell_{H}\right) \omega_{H}-U_{\ell}\left(c_{H}, \ell_{H}\right)\right] y=0, \\
U_{c}\left(c_{L}, \ell_{L}\right) U_{\ell}\left(c_{H}, \ell_{H}\right) \omega_{L}-U_{c}\left(c_{H}, \ell_{H}\right) U_{\ell}\left(c_{L}, \ell_{L}\right) \omega_{H} \geq 0, \\
{\left[U_{c}\left(c_{L}, \ell_{L}\right) U_{\ell}\left(c_{H}, \ell_{H}\right) \omega_{L}-U_{c}\left(c_{H}, \ell_{H}\right) U_{\ell}\left(c_{L}, \ell_{L}\right) \omega_{H}\right]\left[\overline{S C}_{L}-\left(1-\ell_{L}\right)\right]=0,} \\
\overline{S C}_{L}-\left(1-\ell_{L}\right) \geq 0, \\
(1-\gamma) c_{L}+\gamma c_{H}=y, \\
\omega_{L}=F_{1-\ell_{L}}\left[\gamma\left(1-\ell_{H}\right),(1-\gamma)\left(1-\ell_{L}\right)\right], \\
\omega_{H}=F_{1-\ell_{H}}\left[\gamma\left(1-\ell_{H}\right),(1-\gamma)\left(1-\ell_{L}\right)\right],
\end{gathered}
$$

and

$$
y=F\left[\gamma\left(1-\ell_{H}\right),(1-\gamma)\left(1-\ell_{L}\right)\right]
$$

Then, the Lagrangian function associated to the Ramsey problem:

$$
\begin{aligned}
\mathcal{L}= & \varphi \gamma U\left(c_{H}, \ell_{H}\right)+(1-\varphi)(1-\gamma) U\left(c_{L}, \ell_{L}\right)+ \\
& +\lambda^{1}\left\{U_{c}\left(c_{H}, \ell_{H}\right) c_{H} \omega_{H}-U_{\ell}\left(c_{H}, \ell_{H}\right) \omega_{H}\left(1-\ell_{H}\right)-\left[U_{c}\left(c_{H}, \ell_{H}\right) \omega_{H}-U_{\ell}\left(c_{H}, \ell_{H}\right)\right] y\right\}+ \\
& +\lambda^{2}\left[(1-\gamma) c_{L}+\gamma c_{H}-y\right]+ \\
& +\lambda^{3}\left[U_{c}\left(c_{L}, \ell_{L}\right) U_{\ell}\left(c_{H}, \ell_{H}\right) \omega_{L}-U_{c}\left(c_{H}, \ell_{H}\right) U_{\ell}\left(c_{L}, \ell_{L}\right) \omega_{H}\right]+ \\
& +\lambda^{4}\left[S C_{L}-\left(1-\ell_{L}\right)\right]+ \\
& +\lambda^{5}\left[U_{c}\left(c_{L}, \ell_{L}\right) U_{\ell}\left(c_{H}, \ell_{H}\right) \omega_{L}-U_{c}\left(c_{H}, \ell_{H}\right) U_{\ell}\left(c_{L}, \ell_{L}\right) \omega_{H}\right]\left[S C_{L}-\left(1-\ell_{L}\right)\right]
\end{aligned}
$$

The first order conditions with respect to $c_{H}, \ell_{H}, c_{L}, \ell_{L}$, and $\overline{S C}_{L}$ are respectively:

$$
\frac{\partial \mathcal{L}}{\partial c_{H}}=\varphi \gamma U_{c}^{H}+\lambda^{1} A_{0}+\lambda^{2} \gamma+\Delta A_{1}=0
$$

where 


$$
\begin{gathered}
\Delta=\lambda^{3}+\lambda^{5}\left[\overline{S C}_{L}-\left(1-\ell_{L}\right)\right] \\
A_{0}=U_{c c}^{H} \omega_{H} c_{H}+U_{c}^{H} \omega_{H}-U_{\ell c}^{H} \omega_{H}\left(1-\ell_{H}\right)-\left(U_{c c}^{H} \omega_{H}-U_{\ell c}^{H}\right) y,
\end{gathered}
$$

and

$$
\begin{gathered}
A_{1}=U_{c}^{L} U_{\ell c}^{H} \omega_{L}-U_{c c}^{H} U_{\ell}^{L} \omega_{H} . \\
\frac{\partial \mathcal{L}}{\partial \ell_{H}}=\varphi \gamma U_{\ell}^{H}+\lambda^{1} A_{2}-\lambda^{2} \frac{\partial y}{\partial \ell_{H}}+\Delta A_{3}=0
\end{gathered}
$$

where

$$
\begin{aligned}
A_{2}= & \left\{U_{c \ell}^{H} \omega_{H} c_{H}+U_{c}^{H} \frac{\partial \omega_{H}}{\partial \ell_{H}} c_{H}-U_{\ell \ell}^{H} \omega_{H}\left(1-\ell_{H}\right)-U_{\ell}^{H}\left[\frac{\partial \omega_{H}}{\partial \ell_{H}}\left(1-\ell_{H}\right)-\omega_{H}\right]\right. \\
& \left.-\left(U_{c \ell}^{H} \omega_{H}+U_{c}^{H} \frac{\partial \omega_{H}}{\partial \ell_{H}}-U_{\ell \ell}^{H}\right) y-\left(U_{c}^{H} \omega_{H}-U_{\ell}^{H}\right) \frac{\partial y}{\partial \ell_{H}}\right\}
\end{aligned}
$$

and

$$
\begin{gathered}
A_{3}=U_{c}^{L} U_{\ell \ell}^{H} \omega_{L}+U_{c}^{L} U_{\ell}^{H} \frac{\partial \omega_{L}}{\partial \ell_{H}}-U_{c \ell}^{H} U_{\ell}^{L} \omega_{H}-U_{c}^{H} U_{\ell}^{L} \frac{\partial \omega_{H}}{\partial \ell_{H}} \\
\frac{\partial \mathcal{L}}{\partial c_{L}}=(1-\varphi)(1-\gamma) U_{c}^{L}+\lambda^{2}(1-\gamma)+\Delta A_{4}=0 \\
A_{4}=U_{c c}^{L} U_{\ell}^{H} \omega_{L}-U_{c}^{H} U_{\ell c}^{L} \omega_{H} \\
\frac{\partial \mathcal{L}}{\partial \ell_{L}}=(1-\varphi)(1-\gamma) U_{\ell}^{L}+\lambda^{1} A_{5}-\lambda^{2} \frac{\partial y}{\partial \ell_{L}}+\Delta A_{6}+\lambda^{4}+\lambda^{5}\left(U_{c}^{L} U_{\ell}^{H} \omega_{L}-U_{c}^{H} U_{\ell}^{L} \omega_{H}\right)=0
\end{gathered}
$$

where

$$
A_{5}=U_{c}^{H} c_{H} \frac{\partial \omega_{H}}{\partial \ell_{L}}-U_{\ell}^{H}\left(1-\ell_{H}\right) \frac{\partial \omega_{H}}{\partial \ell_{L}}-U_{c}^{H} y \frac{\partial \omega_{H}}{\partial \ell_{L}}-\left(U_{c}^{H} \omega_{H}-U_{\ell}^{H}\right) \frac{\partial y}{\partial \ell_{L}},
$$

and

$$
A_{6}=U_{c \ell}^{L} U_{\ell}^{H} \omega_{L}+U_{c}^{L} U_{\ell}^{H} \frac{\partial \omega_{L}}{\partial \ell_{L}}-U_{c}^{H} U_{\ell \ell}^{L} \omega_{H}-U_{c}^{H} U_{\ell}^{L} \frac{\partial \omega_{H}}{\partial \ell_{L}} .
$$

Finally,

$$
\frac{\partial \mathcal{L}}{\partial \overline{S C}}=\lambda^{4}+\lambda^{5}\left[U_{c}^{L} U_{\ell}^{H} \omega_{L}-U_{c}^{H} U_{\ell}^{L} \omega_{H}\right]=0
$$


To solve for the optimal policies, we follow a two-step procedure:

Step 1: Let us assume first that the minimum wage constraint is binding. In that case we know that:

$$
\begin{array}{cc}
1-\ell_{L}=\overline{S C}_{L} \quad & \lambda^{4}=0 \\
U_{c}^{L} U_{\ell}^{H} \omega_{L}-U_{c}^{H} U_{\ell}^{L} \omega_{H}>0 & \lambda^{3}=0
\end{array}
$$

This implies that $\Delta=0$. Then, substituting into (20), (22):

$$
\begin{gathered}
\varphi \gamma U_{c}^{H}+\lambda^{1} A_{0}+\lambda^{2} \gamma=0 \\
\varphi \gamma U_{\ell}^{H}+\lambda^{1} A_{2}-\lambda^{2} \frac{\partial y}{\partial \ell_{H}}=0 \\
(1-\varphi)(1-\gamma) U_{c}^{L}+\lambda^{2}(1-\gamma)=0
\end{gathered}
$$

From (23) and (24), the following can be obtained:

$$
0=(1-\varphi)(1-\gamma) U_{\ell}^{L}+\lambda^{1} A_{5}-\lambda^{2} \frac{\partial y}{\partial \ell_{L}}
$$

These last four equations jointly with the restriction (9) in the planner's problem and the resource constraint (13) constitute a system of six equations to be solved for $c_{L}, c_{H}, \ell_{L}, \ell_{H}, \lambda^{1}$, and $\lambda^{2}$. After solving this set of equations, we substitute into (10) to check the sign of the inequality. When the sign is positive, it is optimal to set a minimum wage that will be binding. Otherwise, greater welfare is attained under a perfectly competitive labor market . Equilibrium allocations for such a case are computed following directions presented in Step 2.

Step 2: when it is not optimal to set a minimum wage, no worker will be constrained in the labor market. As a result,

$$
\begin{gathered}
1-\ell_{L}<\overline{S C}_{L} \quad \lambda^{4}=0 \\
U_{c}^{L} U_{\ell}^{H} \omega_{L}-U_{c}^{H} U_{\ell}^{L} \omega_{H}=0 \quad \lambda^{3}>0
\end{gathered}
$$

Substituting into the Ramsey problem first order conditions (20), (21) and (23) and rewriting the system we have:

$$
0=\varphi \gamma U_{c}^{H}+\lambda^{1} A_{0}+\lambda^{2} \gamma+A_{1} \Delta
$$




$$
\begin{gathered}
0=\varphi \gamma U_{\ell}^{H}+\lambda^{1} A_{2}-\lambda^{2} \frac{\partial y}{\partial \ell_{H}}+A_{3} \Delta \\
0=(1-\varphi)(1-\gamma) U_{\ell}^{L}+\lambda^{1} A_{5}-\lambda^{2} \frac{\partial y}{\partial \ell_{L}}+A_{5} \Delta
\end{gathered}
$$

and from equation (22) we obtain that:

$$
\Delta=-\frac{1}{A_{4}}\left[(1-\varphi)(1-\gamma) U_{c}^{L}+\lambda^{2}(1-\gamma)\right]
$$

We substitute this expression for $\Delta$ into the three equations above. Then, these three equations, equation (9), the resource constraint (13), and the following condition:

$$
U_{c}^{L} U_{\ell}^{H} \omega_{L}-U_{c}^{H} U_{\ell}^{L} \omega_{H}=0,
$$

have to be solved to find the optimal values for $c_{L}, c_{H}, \ell_{L}, \ell_{H}, \lambda^{1}$, and $\lambda^{2}$. 


\section{References}

[1] Allen, S. (1987): "Taxes, Redistribution, and the Minimum Wage: A Theoretical Analysis", The Quarterly Journal of Economics, vol. 102, pp 477-490.

[2] Askenazy, P. (2003): "Minimum wage, exports and growth" European Economic Review, vol. 47 pp. $147-164$.

[3] Bhaskar, V and T. To (1999): "Minimum Wages for Ronald McDonald monopsonies: A Theory of Monopsonistic Competition", Economic Journal, vol. 109, pp. 190-203.

[4] Boadway, R. and K. Cuff (2001): "A Minimum Wage can be Welfare-Improving and Employment Enhancing" European Economic Review 45 pp. 553-576.

[5] Chari, V.V. and P. Kehoe (1999): "Optimal Fiscal and Monetary Policy", in: John B. Taylor and Michael Woodford, eds., Handbook of Macroeconomics, Vol. 1C, (Elsevier, Amsterdam).

[6] Dickens, R., Machin, S., and A. Manning (1999): "The Effect of Minimum Wages on Employment: Theory and Evidence from Britain", Journal of Labor Economics, vol.17, pp. 1-22.

[7] Dolado, J., Kramarz F., Machin S., Manning. A., Margolis, D. and C. Teulings (1996): "The Economic Impact of Minimum Wages in Europe", Economic Policy, vol. 17, pp. 317-373.

[8] Dolado, J., Felgueroso, F., and J.F. Jimeno (2000): "The Role of the Minimum Wage in the Welfare State: An Appraisal", Swiss Journal of Economics and Statistics,vol. 136, pp. 1-33.

[9] Cahuc, P. and P. Michel (1996): "Minimum Wage, Unemployment and Growth", European Economic Review, vol. 40, pp. 1463-1482.

[10] Drèze, J. and C. Gollier (1993): "Risk Sharing on the Labour Market and Second-Best Wage Rigidities", European Economic Review, vol. 37, pp. 1457-1482.

[11] Eurostat (1997): "Minimum wages 1997. A Comparative Study". Eurostat (Luxembourg).

[12] Gorostiaga, A. (2003): "Should Fiscal Policy Be Different in a Non-Competitive Framework". Journal of Monetary Economics, vol. 50/6, pp. 1311-1331. 
[13] Gorostiaga, A. (2005): "Optimal Fiscal Policy with Rationing in the Labor Market". Topics in Macroeconomics, vol. 5 No. 1, Article 17.

[14] Guesnerie, R and K. Roberts (1987): "Minimum Wage Legislation as a Second Best Policy", European Economic Review, vol. 31, pp. 490-498.

[15] Lang, K. and S. Kahn (1998): "The Effect of Minimum-Wage Laws on the Distribution of Employment: Theory and Evidence", Journal Public Economics, vol. 69, pp. 67-82.

[16] Lucas, R. and N. Stokey (1983): "Optimal Fiscal and Monetary Policy in an Economy without Capital", Journal of Monetary Economics, vol. 12, pp. 55-93.

[17] Malinvaud, E. (1977): The Theory of Unemployment Reconsidered. Oxford: Basil Blackwell.

[18] Marceau, N. and R. Boadway (1994): "Minimum Wage Legislation and Unemployment Insurance as Instruments for Redistribution", Scandinavian Journal of Economics, vol. 96 , pp. $67-81$.

[19] OECD (1998). "Making the Most of the Minimum: Statutory Minimum Wages, Employment and Poverty". Employment Outlook, OECD. Paris.

[20] Ramsey, F. (1927): "A Contribution to the Theory of Taxation", Economic Journal, vol. 37, pp.47-61.

[21] Rebitzer, J. and L. Taylor (1995) "The Consequences of Minimum Wage Laws: Some New Theoretical Ideas", Journal of Public Economics, vol. 56, pp. 245-255.

[22] Rubio-Ramírez, J.F. (2000): "Redistribution and Fiscal Policy". University of Minnesota and Federal Reserve Bank of Minneapolis. Mimeo.

[23] Saint Paul, G.(1994): "Do Labor Market Rigidities Fulfill Distributive Objectives?", IMF Staff Papers, vol 41, pp.624-642.

[24] Sampson, A and R. Simmons (2002): "The minimum wage in an adverse selection economy", Oxford Economic Papers, vol. 54, pp. 150-159

[25] Schmitt-Grohé, S. and M. Uribe (2004): "Optimal Fiscal and Monetary Policy Under Sticky Prices", Journal of Economic Theory, vol. 114, pp. 198-230.

[26] Swinnerton, K. (1996): "Minimum Wages in an Equilibrium Search Model with Diminishing Returns to Labor in Production", Journal of Labor Economics, vol. 14, pp. $340-355$. 

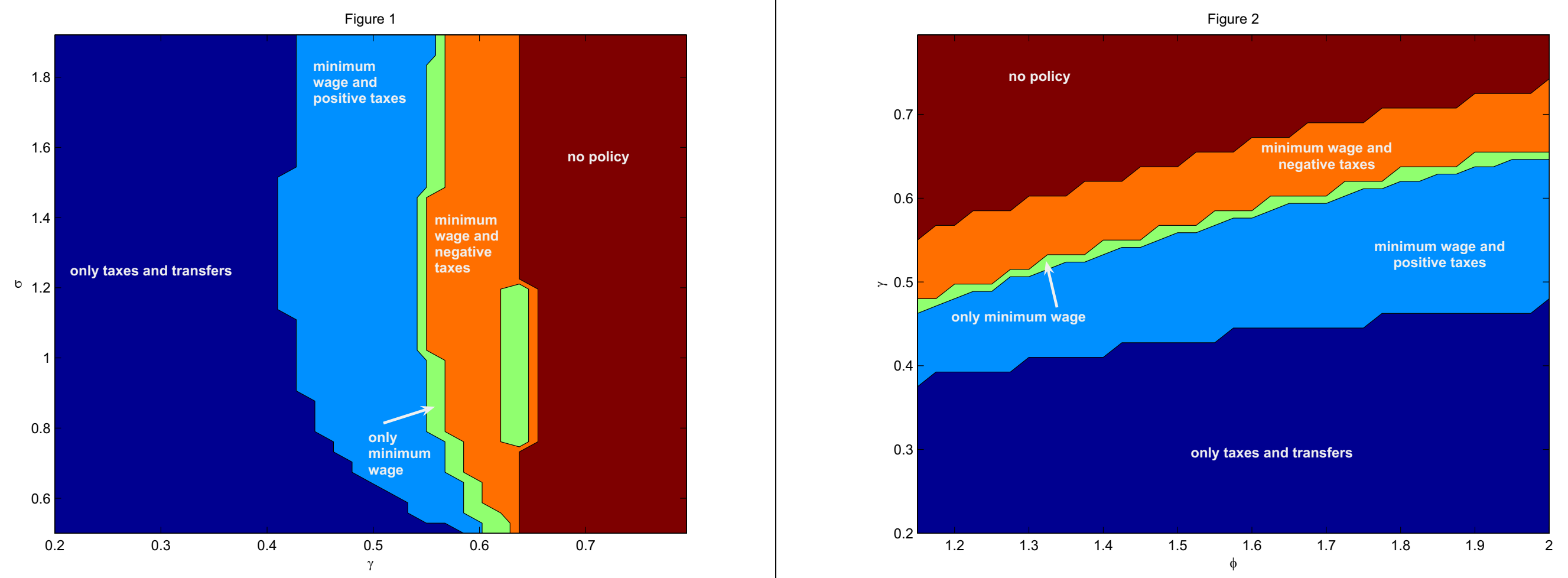
Figure 3

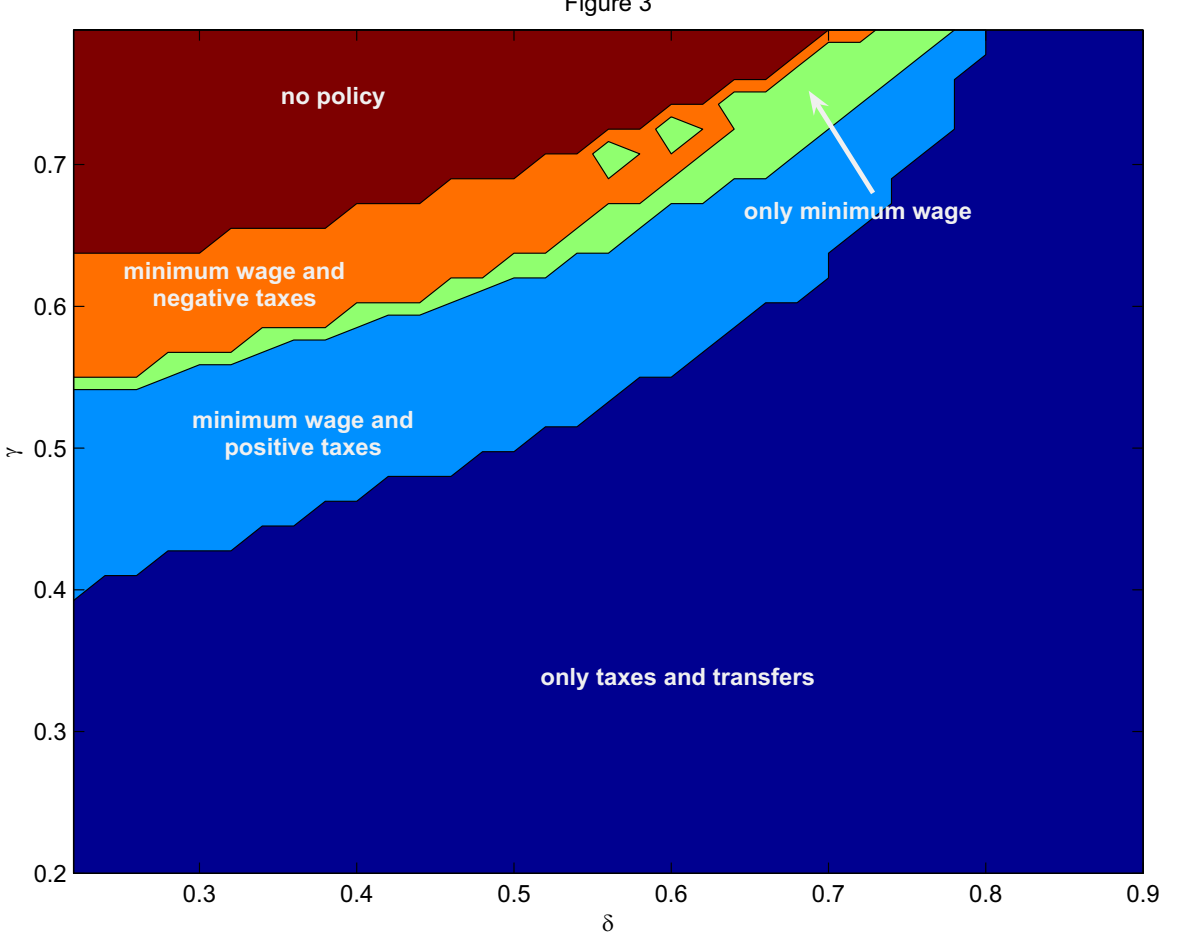

Figure 4

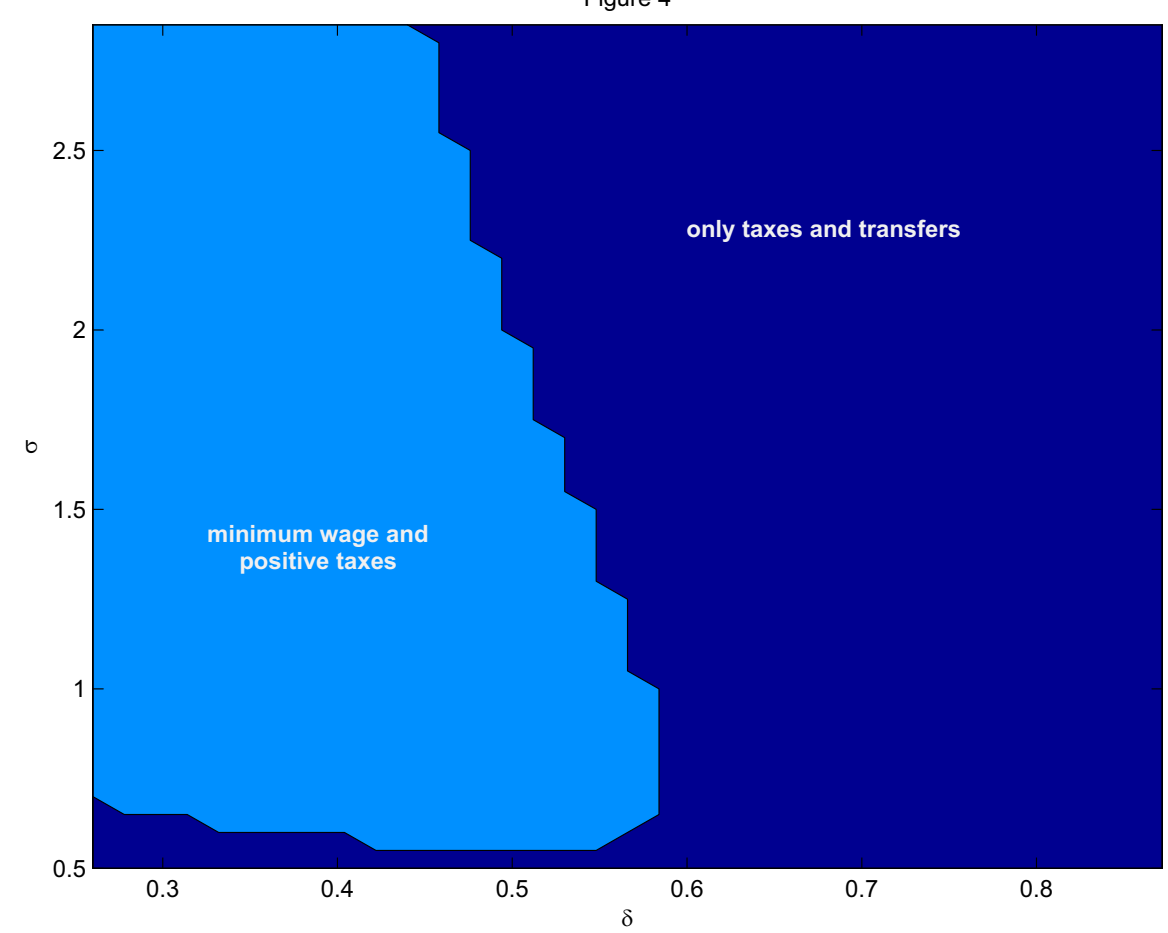


Figure 5

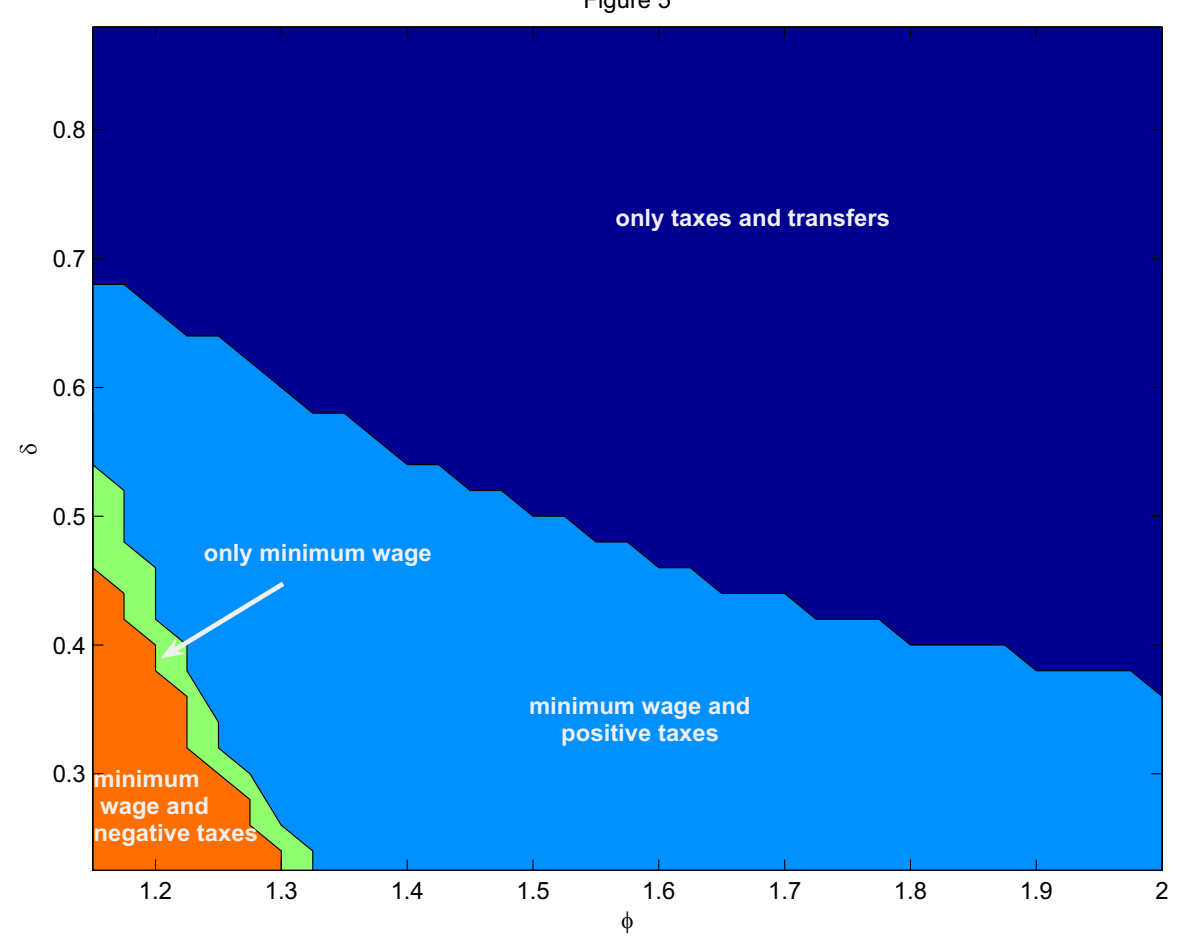

Figure 6

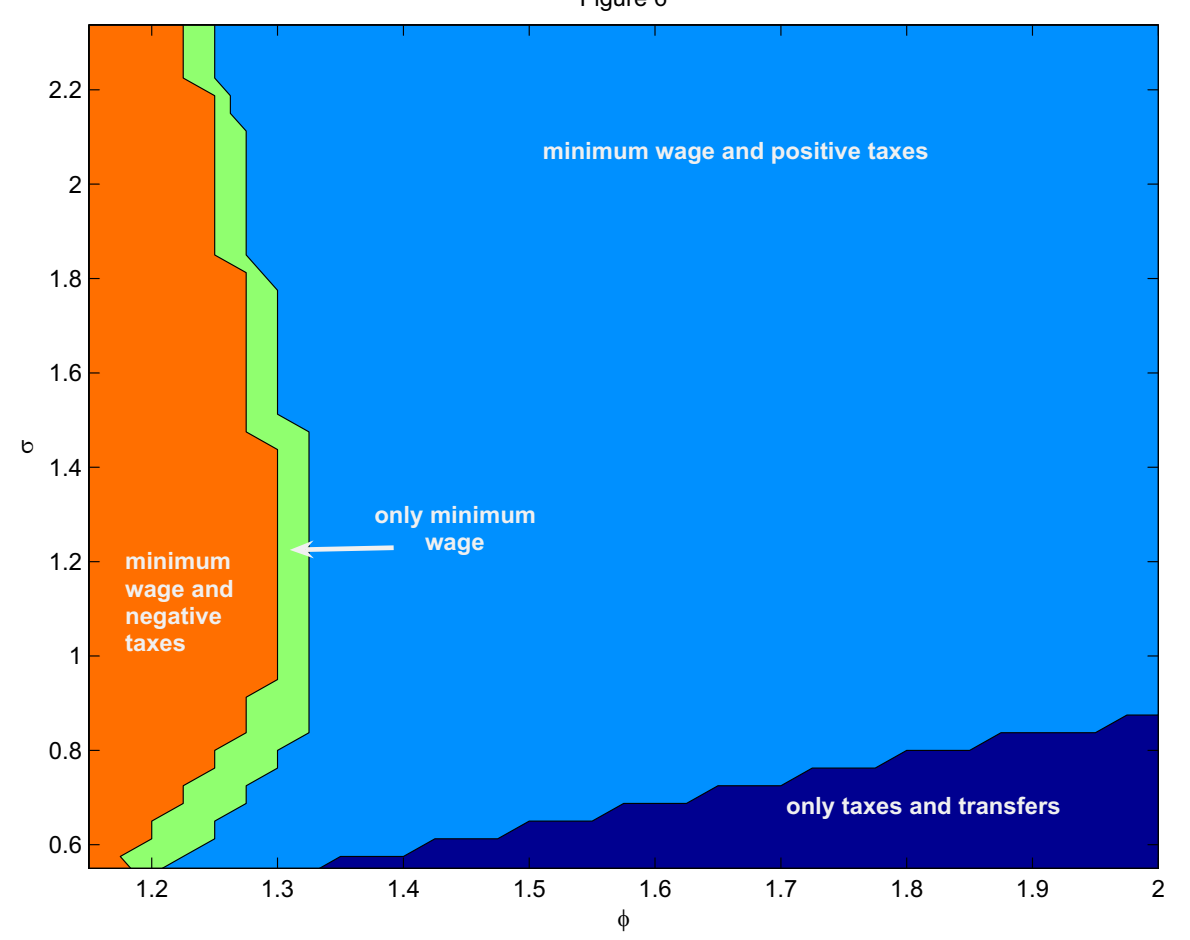

